\title{
On mentoring in nursing academia - Le mentorat en milieu universitaire en sciences infirmières
}

Jacinthe I. Pepin

Université de Montréal, jacinthe.pepin@umontreal.ca

Florence Myrick

amyrick@ualberta.ca

Follow this and additional works at: https://qane-afi.casn.ca/journal

Part of the Scholarship of Teaching and Learning Commons

\section{Recommended Citation}

Pepin, Jacinthe I. and Myrick, Florence (2017) "On mentoring in nursing academia - Le mentorat en milieu universitaire en sciences infirmières," Quality Advancement in Nursing Education - Avancées en formation infirmière: Vol. 3: Iss. 2, Article 1.

DOI: https://doi.org/10.17483/2368-6669.1143

This Editorial is brought to you for free and open access by Quality Advancement in Nursing Education - Avancées en formation infirmière. It has been accepted for inclusion in Quality Advancement in Nursing Education - Avancées en formation infirmière by an authorized editor of Quality Advancement in Nursing Education - Avancées en formation infirmière. 


\section{On mentoring in nursing academia}

Increasingly, in academia, mentoring is recognized as being among the best practices with which to attract faculty members and to ensure their successful careers. Mentoring is perceived as a process of generating positive effects on quality education programs and on successful competitive research proposals. In nursing academia in particular, formal mentoring programs are designed and implemented in many countries at the faculty, university, regional as well as national levels. Mentoring within the professional discipline of nursing can be considered twofold, vis-a-vis tenure-track positions as well as clinical educators. More recently, evidence has emerged concerning the effectiveness of mentoring interventions and specific program plans. Recently, Nowell and colleagues $(2015,2017)$ conducted a systematic review to critically appraise this evidence and to report on outcomes regarding mentees, mentors, and nursing education institutions.

In this issue of Quality Advancement in Nursing Education / Avancées en formation infirmière, two articles which reflect mentoring in nursing academia are published: one describing a graduate students' designed mentoring program focused on networking within and outside of one's faculty (Bell et al.), and the second one examining the potential of faculty learning communities to promote scholarship and collegiality, for example, with pre-tenured members (Yonge et al.). Also, our current interview describes the intent, design, and lessons learned concerning the annual Canadian Doctoral Nursing Network Conference, convened to create a community of support among doctoral students with the participation and contribution of scholars from coast to coast. These three endeavours could be said to be the tip of the iceberg with regard to the mentoring undertakings occurring within Canadian nursing academia in the last five years. Other examples encompass domain specific networks such as the Doctoral Student Network created in 2013 by the Canadian Association of Nurses in Oncology (CANO), and interuniversity deans and director's collaboration to obtain recurrent scholarship support attracting doctoral students or to facilitate doctoral students as they transition to academic careers.

While formal evaluation of mentoring programs is vital to building stronger evidence required for further advancement (Nowell et al., 2017), we suggest that the goals of mentoring be closely examined for their alignment with the nature of nursing academia. Systemic programs pursue goals such as "to accelerate the development of the next generation of academic nurse leaders" (p. 340). The more specific goals revolve, understandably, around developing the teaching scholarship of new faculty and increasing their research success and productivity. The success of individual nursing academics in the pursuit of quality education, research, and scholarship, appears then to be on a fruitful path. As part of the mentoring process, we would also like to recommend considering the collective goals for the discipline of nursing, specifically fostering the work of future "nurse philosophers and also some philosophizing on the part of the nurse researchers", as Donaldson and Crowley suggested in 1978 (p. 114). Equally, consider the question, how will nursing schools and faculty engage the next generation of leaders in positioning themselves in future university paradigms? Consider also mentoring for a sense of collegiality and politics. Adopting a larger lens might indeed bring nursing academia even further forward.

Equally informative are the remaining articles, published in this issue, which revisit problem-based learning (Mirza et al.; Pierazzo et al.) and population health promotion practicums (Laliberte et al.). 


\section{Le mentorat en milieu universitaire en sciences infirmières}

Dans le milieu universitaire, le mentorat est de plus en plus reconnu comme étant l'une des meilleures pratiques pour intéresser des personnes à la carrière professorale et en assurer le succès. Le mentorat est perçu comme ayant des effets positifs sur la qualité des programmes de formation et sur la réussite de demandes compétitives de subventions de recherche. En sciences infirmières, des programmes formels de mentorat sont notamment conçus et implantés dans les facultés et écoles, dans les universités, aux plans régional et national, dans de nombreux pays. Le mentorat dans la discipline professionnelle infirmière comporte deux volets, celui des professeures de carrière et celui des formatrices cliniques. Le nombre de publications rapportant des résultats quant à l'efficacité des interventions et programmes spécifiques de mentorat a récemment augmenté. Ainsi, Nowell et coll. $(2015 ; 2017)$ ont effectué une revue systématique des écrits afin d'examiner ces résultats de manière critique et de mettre en évidence ceux qui concernent les mentorées, les mentors et les établissements de formation en sciences infirmières.

Le présent numéro de Quality Advancement in Nursing Education / Avancées en formation infirmière comprend deux articles qui traitent du mentorat en milieu universitaire en sciences infirmières : un décrivant un programme de mentorat conçu par des étudiantes des cycles supérieurs, centré sur le réseautage dans son milieu académique et en dehors de celui-ci (Bell et coll.); l'autre se penchant sur le potentiel qu'ont les communautés apprenantes de professeures de promouvoir le scholarship et la collégialité, par exemple, chez les membres du corps professoral n'ayant pas encore demandé leur agrégation (Yonge et coll.). De plus, notre entrevue présente la Canadian Doctoral Nursing Networking Conference (Conférence canadienne de réseautage au doctorat en sciences infirmières), ses objectifs, sa conception et les leçons tirées. Cette Conférence a été organisée afin de créer une communauté de soutien entre étudiantes au doctorat, avec la participation et l'appui d'universitaires de partout au pays. Ces trois initiatives ne représentent qu'une infime partie des démarches de mentorat entreprises dans les programmes de formation en sciences infirmières au Canada, en particulier au cours des cinq dernières années. D'autres exemples comprennent des réseaux de soutien spécifiques au domaine, comme le Réseau des étudiantes au doctorat et post-doctorat, créé en 2013 par l'Association canadienne des infirmières en oncologie (ACIO), ou encore une collaboration interuniversitaire entre doyens et directeurs pour obtenir des bourses récurrentes de manière à attirer des étudiantes au doctorat et faciliter leur transition vers une carrière académique.

Bien que l'évaluation formelle des programmes de mentorat soit vitale pour constituer les bases nécessaires à leur avancement (Nowell et coll., 2017), nous suggérons que les objectifs du mentorat soient réexaminés afin de les aligner encore plus étroitement à la nature du monde universitaire en sciences infirmières. Les programmes systémiques de mentorat visent des objectifs tels que «l'accélération du déploiement de la prochaine génération de leaders infirmières universitaires » (trad. libre, p. 340). Les objectifs plus spécifiques portent évidemment sur le développement du scholarship en formation des nouvelles professeures et l'amélioration de leur productivité et de la réussite de leurs recherches. Individuellement, les nouveaux professeurs en sciences infirmières semblent donc sur la voie de la réussite pour leurs enseignements et leurs recherches de qualité. Nous recommandons cependant de viser des objectifs collectifs du mentorat en milieu universitaire, pour le développement de la discipline infirmière, plus particulièrement, le soutien aux travaux de futures «infirmières philosophes, ainsi qu'à une certaine dose de philosophie de la part des infirmières chercheures », comme le suggéraient Donaldson et Crowley en 1978 (trad. libre, p. 114). Il faut également se poser la 
question : comment les écoles et facultés de sciences infirmières inciteront-elles la prochaine génération de leaders à se positionner dans les futurs paradigmes et orientations des universités? Il importe donc de penser au mentorat aussi pour développer un sens de la collégialité et du politique. L'adoption d'une perspective plus large pourrait effectivement faire avancer encore davantage le milieu universitaire en sciences infirmières.

D'autres articles d'un aussi grand intérêt s'ajoutent à ce numéro et incitent à réexaminer l'apprentissage par problèmes (Mirza et coll.; Pierazzo et coll.) et les stages de promotion de la santé publique (Laliberté et coll.).

Cordialement / Respectfully,

Corédactrices en chef, Co-Editors-in-Chief,

Jacinthe Pepin, inf. Ph.D., Faculté des sciences infirmières, Université de Montréal

Florence Myrick, RN, Ph. D., Faculty of Nursing, University of Alberta

\section{References / Références}

Donaldson, S., \& Crowley, D. (1978). The discipline of nursing. Nursing Outlook, 26(2), 113120.

Nowell, L., White, D. E, Mrklas, K., \& Norris, J. M. (2015). Mentorship in nursing academia: a systematic review protocol. Systematic Reviews, 4. https://doi.org/10.1186/s13643-0150007-5

Nowell, L., Norris, J., Mrklas, K., \& White, D. (2017). A literature review of mentorship programs in academic nursing. Journal of Professional Nursing. 33, 334-344. https://doi.org/10.1016/j.profnurs.2017.02.007 